\title{
Evaporation model of a single hydrocarbon fuel droplet due to ambient turbulence at intermediate Reynolds numbers
}

\author{
J.-S. Wu ${ }^{\text {a,* }}$ K.-H. Hsu ${ }^{\text {a }}$, P.-M. Kuo ${ }^{\text {b }}$, H.-J. Sheen ${ }^{b}$ \\ a Department of Mechanical Engineering, National Chiao-Tung University, 1001 Ta-Hsueh Road, Hsinchu 30050, Taiwan \\ ${ }^{\mathrm{b}}$ Institute of Applied Mechanics, National Taiwan University, Taipei 10670, Taiwan
}

Received 6 March 2003; received in revised form 21 May 2003

\begin{abstract}
A general evaporation model of a single liquid fuel droplet under various ambient turbulence conditions at intermediate droplet Reynolds numbers is proposed combining the current and previous experimental results. Extensive evaporation experiments of single fuel droplets are conducted at room temperature in quasi-laminar and turbulent environments at Reynolds numbers in the range of 72-333. Tested hydrocarbon fuels include by pentane, hexane, heptane, octane and decane. Flow fields, behind a perforated plate or a circular disk in a vertical low-speed wind tunnel, provide the required ambient turbulent environments for droplet evaporation experiments. The ambient turbulence intensities vary in the range of $1-60 \%$ and the integral length scales in the range of $0.5-20$ times of the initial droplet diameter. Results show that normalized evaporation rate $\left(K / K_{\mathrm{L}}\right)$ decreases with increasing effective Damköhler number $\left(0.0001<D a_{\mathrm{v}}<0.1\right)$ and is approximately equal to unity with further increasing Damköhler number $\left(0.1<D a_{\mathrm{v}}<1\right)$.

(C) 2003 Elsevier Ltd. All rights reserved.
\end{abstract}

\section{Introduction}

Due to its importance in spray combustion, liquid droplet evaporation has been thoroughly studied in the past, for detailed reviews see Faeth [1], Sirignano [2], and references cited therein. Most previous experimental and numerical studies of the evaporating process of a single liquid droplet (e.g., references in Refs. [1,2]) were carried out either in laminar natural convection or in laminar forced convection neglecting the importance of ambient turbulence, as pointed out by Golkap et al. [3] and $\mathrm{Wu}$ et al. [4]. Relatively few researches (e.g. [3-6]) have studied the effects of ambient turbulence on droplet vaporization in forced convection. Conclusions from these studies generally agreed that ambient turbulence speeds up the evaporation process of a single liquid droplet in forced convection. However, the studies on droplet evaporation either emphasized on the influence of turbulence inten-

\footnotetext{
${ }^{*}$ Corresponding author. Tel.: +886-3-573-1693; fax: +886-3572-0634.

E-mail address: chongsin@cc.nctu.edu.tw (J.-S. Wu).
}

sities by largely neglecting the effects of length scales [6] or the combined effects of both length scales and turbulence intensities were not clearly identified $[3,5,6]$. In addition, coupled Reynolds numbers and fuel effects were not discussed in detail either. Among these studies, Gokalp et al. [3] pioneered to propose an effective vaporization Damköhler number, $D a_{\mathrm{v}}=T_{\text {ed }} / T_{\mathrm{v}}$, where $T_{\text {ed }}$ is the turbulence eddy time scale based on the initial droplet size and $T_{\mathrm{v}}$ is the vaporization time scale based on the film theory, to roughly describe the combined effects of fuel and ambient turbulence properties on droplet evaporation. However, a systematic study revealing the correlation between $D a_{\mathrm{v}}$ and droplet evaporation rate was not available until the work of Wu et al. [4].

Wu et al. [4] has successfully characterized the droplet evaporation (at room temperature) through the effective vaporization Damköhler number by combining the effects of length scales and turbulence intensities of ambient turbulence, and the effects of fuels (heptane and decane) at droplet Reynolds number of 100. Resulting correlation stated that the normalized evaporation rate is proportional to $D a_{\mathrm{v}}^{-0.115}$ for $D a_{\mathrm{v}}$ in the range of 0.0001-0.1. 


\begin{tabular}{|c|c|c|c|}
\hline \multicolumn{4}{|c|}{ Nomenclature } \\
\hline$d$ & instantaneous fuel droplet diameter & $R e_{\ell}$ & Turbulence Reynolds number based on in- \\
\hline$D a_{\mathrm{v}}$ & vaporization Damköhler number & & tegral length scale $\left(=u^{\prime} \ell / v\right)$ \\
\hline$D_{1}$ & diameter of the circular pipe & $U$ & mean streamwise velocity \\
\hline$D_{3}$ & diameter of the contraction exit & $U_{\mathrm{c}}$ & mean streamwise velocity at the center of the \\
\hline$K_{\mathrm{L}}$ & droplet vaporization constant under laminar & & pipe or the contraction exit \\
\hline$K_{0}$ & $\begin{array}{l}\text { convection conditions } \\
\text { droplet vaporization constant under stag- }\end{array}$ & $u$ & $\begin{array}{l}\text { instantaneous streamwies fluctuating veloc- } \\
\text { ity }\end{array}$ \\
\hline & nant conditions & $u^{\prime}$ & r.m.s streamwise fluctuating velocity \\
\hline$K$ & droplet vaporization constant under turbu- & $V$ & mean cross-streamwise velocity \\
\hline & lent convection conditions & $v^{\prime}$ & radial r.m.s fluctuating velocity \\
\hline$L$ & depth from the test point & $x$ & streamwise coordinate \\
\hline$\ell$ & turbulence integral length scale & $y$ & cross-streamwise coordinate \\
\hline$R e_{\mathrm{d}}$ & $\begin{array}{l}\text { Reynolds number based on the droplet dia- } \\
\text { meter }\left(R e_{\mathrm{d}}=U d / v\right)\end{array}$ & & \\
\hline
\end{tabular}

Therefore, the present experimental investigation was undertaken to extend the understanding of these effects by considering broader test conditions $\left(R e_{\mathrm{d}}=72-333\right.$; more hydrocarbon fuels) and reevaluate the validity of the proposed evaporation model by $\mathrm{Wu}$ et al. [4] in describing droplet evaporation at room temperature.

\section{Experimental methods}

\subsection{Apparatus}

The apparatus included an up-flowing air wind tunnel with the test liquid droplet suspended near the axis at the downstream end of the wind tunnel, where the detailed information was provided in Ref. [4]. This configuration provided both satisfied quasi-laminar (with the contraction nozzle) and typical turbulent (without the contraction nozzle) pipe flow properties for droplet evaporation experiments. To generate the required turbulent intensities and length scales, a circular disk plate was placed at different locations within the vertical long pipe to create a nearly homogeneous turbulent environment with $u^{\prime} / U=1-60 \%$ and $\ell=3.6-38 \mathrm{~mm}$ at the test section [4]. To obtain smaller integral length scales in this study, a perforated plate was placed in the contraction nozzle followed by a constant-diameter mixing tube with different lengths (Fig. 1). By changing plates with different porosities in the contraction and mixing tubes, we are able to achieve $\ell=1.1 \mathrm{~mm}$, which is either smaller or comparable with the size of the liquid droplet.

\subsection{Instrumentation}

Description of instrumentation about the current research can be found in Ref. [4] and will not be de- scribed in detail for brevity. Free-stream flow fields in the test section were measured using a traverible laser velocimeter with $\mathrm{MgO}$ as the seeding particles. Experimental uncertainties ( $95 \%$ confidence) largely were controlled by sampling limitations to yield the following values for mean velocities of $0.5-3 \mathrm{~m} / \mathrm{s}$ : mean velocities less than $5 \%$, fluctuating velocities less than $10 \%$, and temporal power spectrum less than $20 \%$. A digital CCD camera was used to record the droplet images using shadowgraph technique. Concepts of equivalent spherical

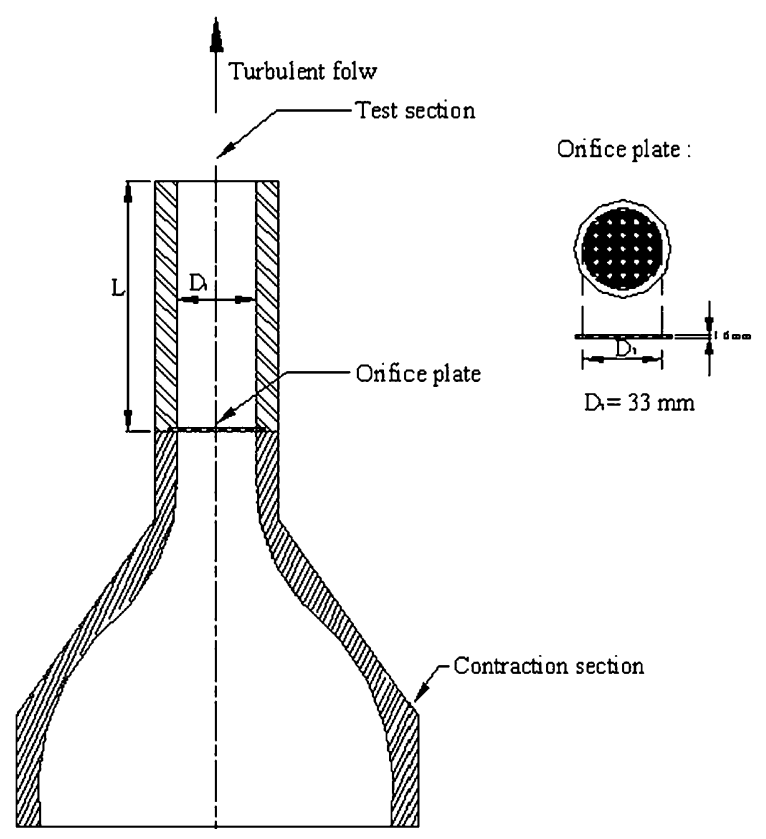

Fig. 1. Schematic diagram of turbulence generator using perforated plate with circular tube and contraction. 
to ellipsoidal volume were used to compute the droplet diameter. Data were obtained by averaging over $3-5$ times of experiments to reduce the experimental uncertainties. Estimated experimental uncertainties $(95 \%$ confidence) for droplet diameter was less than $3 \%$.

\subsection{Test conditions}

Tested droplet Reynolds numbers are in the range of 72-333 to more or less represent typical flow conditions in sprays. Droplet sizes range from 1.5 to $2.5 \mathrm{~mm}$. Typical test conditions generated by the perforated plate for suspended liquid droplets in turbulent environments with $\ell / d \approx 0.44-1.47$ and $u^{\prime} / U_{c} \approx 4-40 \%\left(R e_{\mathrm{d}}=240\right.$ 333). Other test conditions using the circular disk are not listed here for brevity and can be found in Ref. [4]. Mean streamwise velocities at the axis of the tunnel were in the range of $0.5-2.5 \mathrm{~m} / \mathrm{s}$ depending on the Reynolds numbers and droplet size. As in previous study [4], the ambient flow properties were averaged over the central region of the tunnel exit, e.g., $0.1 \leqslant y / D_{1} \leqslant 0.1$. Resulting isotropic values in fluctuating velocity, $u^{\prime} / v^{\prime}$, were in the range of $0.82-1.41$, which represented that the isotropic turbulence approximation was valid. Streamwise integral length scales were found using Taylor's hypothesis, $\ell=U \tau_{u}$, where $\tau_{u}$ was fitted from the measurements of temporal power spectrum of velocity fluctuations at very low frequency and turbulence intensity, assuming isotropic turbulence [4]. Resulting ratios of streamwise integral length scale to droplet diameter were thus in the range $0.44-20$, which is wider than the previous study [4]. Moreover, the turbulence Reynolds numbers, $R e_{\ell}$, ranged 15-280, which were definitely representative of fully developed turbulent flows as discussed by Tennekes and Lumley [7]. Five liquid fuels, including pentane, hexane, heptane, octane and decane were used as the test droplets at room temperature.

In summary, the turbulent flow properties at the exit of the tunnel with a perforated plate in addition to those established previously [4] are nearly isotropic and homogeneous $\left(u^{\prime} / U_{c}=1-60 \%, \ell / d=0.44-20\right)$ in a region of $24 \times 24 \times 30 \mathrm{~mm}$ near the axis. This region is reasonably uniform for further droplet evaporation measurements discussed later.

\section{Droplet evaporation}

\subsection{Droplet evaporation in quasi-laminar environments}

As shown previously [4], the quasi-laminar flow conditions were obtained with the contraction nozzle at the end of the wind tunnel. In addition to the data presented in Ref. [4], we have conducted droplet evaporation experiments by including more hydrocarbon

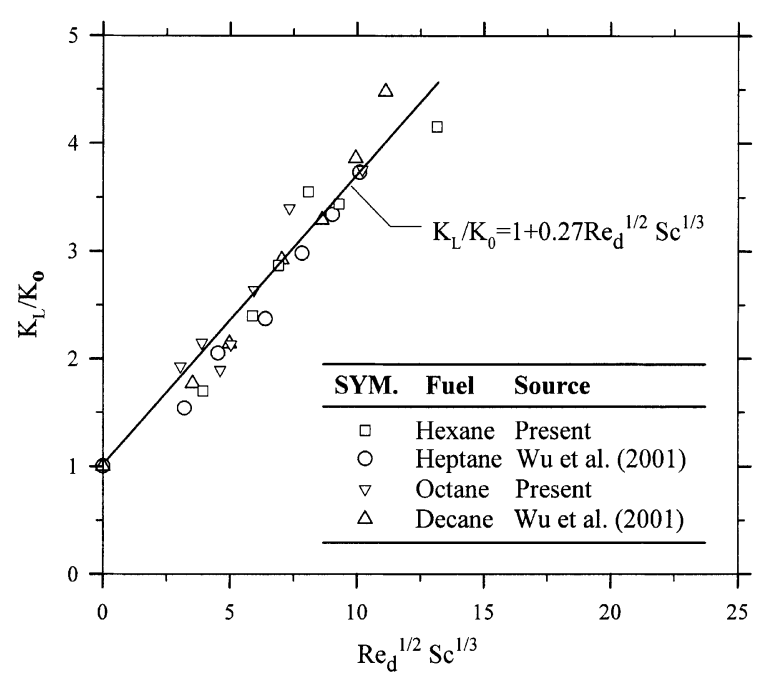

Fig. 2. Measured Frössling correlation for hexane, heptane, octane and decane fuel droplets at intermediate Reynolds numbers.

fuels (hexane and octane) to test if the Frossling correlation is suitable for describing the droplet evaporation in quasi-laminar environments. Results of normalized evaporation rate, $K_{\mathrm{L}} / K_{0}$, as a function of non-dimensionless number, $R e_{\mathrm{d}}^{1 / 2} S c^{1 / 3}$, are illustrated in Fig. 2 including hexane, heptane, octane and decane hydrocarbon fuels for Reynolds number in the range of 60500. Previous data of $\mathrm{Wu}$ et al. [4] are also included in the figure for completeness. Results show the Frossling correlation, $K_{\mathrm{L}} / K_{0}=1+F_{0} R e_{\mathrm{d}}^{1 / 2} S c^{1 / 3}$, are reasonably well with $F_{0}$ fitted as 0.27 , which is close to the value of 0.276 obtained by Frössling [8]. Details of parameter definition and source of physical properties can be found in Ref. [4] and are not repeated here for brevity. Nevertheless, the absolute data of evaporation rate for heptane and decane obtained in the current study for quasi-laminar flow condition are compatible with those of Gokalp et al. [3].

\subsection{Droplet evaporation in turbulent environments}

In the current study, we have measured the droplet evaporation in turbulent environments with normalized integral length scale $\ell / d=0.44-20$ at $R e_{\mathrm{d}}=72-333$ with approximately the same range of turbulence intensities (1-60\%). Droplet Reynolds numbers is varied by carefully adjusting the size of tested liquid droplet (1.5-2.5 $\mathrm{mm}$ ) and speed of the wind tunnel $(0.5-2 \mathrm{~m} / \mathrm{s})$. Wu et al. [4] have shown that, for heptane and decane fuels with $\ell / d=2-20$ and $R e_{\mathrm{d}}=100$, an effective vaporization Damköhler number $\left(D a_{\mathrm{v}}\right)$ can be used to reasonably correlate the effects of the ambient turbulence on the liquid droplet evaporation at these conditions. Note that 
this effective vaporization Damköhler number is defined as the ratio of turbulence-eddy time scale to the evaporation time scale based on thin film theory $[3,4]$. However, it is uncertain that if the proposed correlation in Ref. [4] can be extended to other hydrocarbon fuels at intermediate droplet Reynolds numbers for single droplet evaporation. Therefore, it is interesting to reevaluate this correlation using the new measurements including more fuels and wider ranges of droplet Reynolds numbers. Detailed procedures of computing related properties and parameters have been demonstrated in Ref. [4] and are not repeated here for brevity.

Fig. 3 illustrates the normalized evaporation rate constant $\left(K / K_{\mathrm{L}}\right)$ as a function of the Damköhler number $\left(D a_{\mathrm{v}}\right)$, which extends over four decades $(0.0001-1)$, for various hydrocarbon fuels and Reynolds numbers in the range of 72-333. Previous results of droplet evaporation for heptane and decane fuels with $\ell / d=2-20$ and $R e_{\mathrm{d}}=100$ [4] also have been included in the figure for completeness. In general, $K / K_{\mathrm{L}}$ correlates reasonable well with $D a_{\mathrm{v}}$ with two distinguished regimes. One is with turbulence effect $\left(0.0001<D a_{\mathrm{v}}<0.1\right)$ where $K / K_{\mathrm{L}}$ decreases with increasing $D a_{\mathrm{v}}$, while another is without turbulence effect $\left(0.1<D a_{\mathrm{v}}<1\right)$. For very small

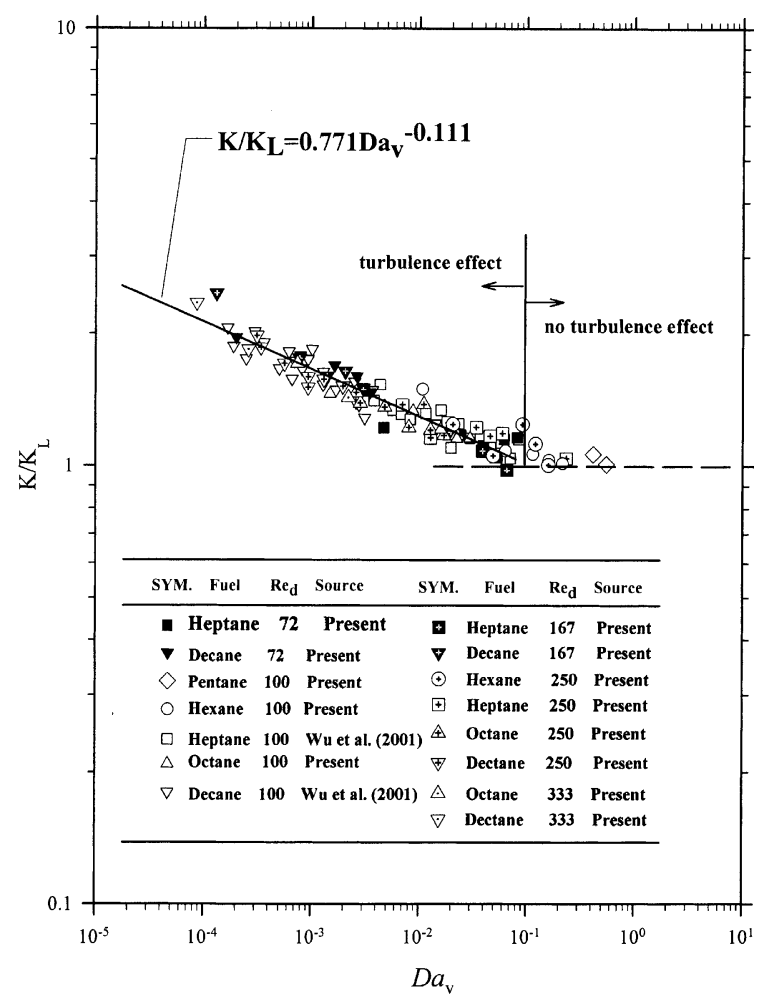

Fig. 3. Normalized evaporation rate as a function of effective vaporization Damkholer number at Reynolds numbers in the range of 72-333.
Damköhler number cases ( $\left.D a_{\mathrm{v}} \approx 0.0001\right)$, the increase of droplet evaporation rate due to the ambient turbulence can be as high as $150 \%$, as shown in Fig. 3. However, for large Damköhler number cases $\left(0.1<D a_{\mathrm{v}}<1\right)$, the effect of the ambient turbulence is found to be practically negligible considering the experimental uncertainties. The correlation formula for the regime with turbulence effects can be expressed as,

$\frac{K}{K_{\mathrm{L}}}=0.771 D a_{\mathrm{v}}^{-0.111} \quad$ for $0.0001 \leqslant D a_{\mathrm{v}} \leqslant 0.1$.

Comparing the current value of pre-exponent factor (0.771) with previous value $(0.741)$ [4], the change is relatively small even with much larger database in the current study. This study nevertheless validates previous proposed correlation with slight changes. Thus, for a single hydrocarbon liquid droplet in turbulent environments at intermediate Reynolds numbers, its evaporation rate can be easily computed using the above proposed correlation once we know the droplet Reynolds numbers and evaporation rate in laminar.

\section{Conclusions}

The evaporation rate of single suspended liquid fuel droplets, including hexane, heptane, octane and decane fuels, in quasi-laminar and turbulent environments was studied experimentally at intermediate droplet Reynolds numbers $\left(R e_{\mathrm{d}}=72-333\right)$ at room temperature. The turbulent environments involved conditions near the exit of a vertical and open-air wind tunnel with nearly isotropic and homogeneous turbulence properties using either a circular disk or a perforated plate in the wind tunnel. The testing turbulent flow conditions include turbulence intensities of roughly $1-60 \%$ and $\ell / d$ in the range $0.44-20$. Experimental results show that two distinct regimes for normalized evaporation rate are found. One is with turbulence effects $\left(K / K_{\mathrm{L}} \propto D a_{\mathrm{v}}^{-0.111}\right)$ for $0.0001<D a_{\mathrm{v}}<0.1$ and another is without turbulence effects $\left(K / K_{\mathrm{L}} \sim 1\right)$ for $0.1<D a_{\mathrm{v}}<1$.

\section{Acknowledgements}

The authors would like to express their sincere thanks to the financial support by the National Science Council of TAIWAN under Grant NSC-89-2212-E-009034 and NSC-90-2212-E-009-012.

\section{References}

[1] G.M. Faeth, Evaporation and combustion of sprays, Prog. Energy Combust. Sci. 9 (1983) 1-76. 
[2] W.A. Sirignano, Fuel droplet vaporation and spray combustion theory, Prog. Energy Combust. Sci. 9 (1983) 291322.

[3] I. Gokalp, C. Chauveau, O. Simon, X. Chesneau, Mass transfer from liquid fuel droplets in turbulent flow, Combust. Flame 89 (1992) 286-298.

[4] J.-S. Wu, Y.-J. Liu, H.-J. Sheen, Effects of ambient turbulence and fuel properties on the evaporation rate of single droplets, Int. J. Heat Mass Transfer 44 (2001) 4593-4603.

[5] N. Hiromitsu, O. Kawaguchi, Influence of flow turbulence on the evaporation rate of a suspended droplet in a hot air flow, Heat Transfer-Jpn. Res. 24 (1997) 689700.

[6] P.R.A. Yearling, Experimental Determination of Convective Heat and Mass Transfer Rates from Single Evaporating Liquid Droplets in a Turbulent Air Flow, PhD Thesis, North Carolina State University, Raleigh, North Carolina, USA, 1995.

[7] H. Tennekes, J.L. Lumley, A First Course in Turbulence, MIT Press, Cambridge, MA, 1972, pp. 113-124.

[8] N. Frössling, Gerlands Gerlands Beitrage zur Geophysik 52 (1938) 170-175. 